\title{
Risk indices predicting graft use, graft and patient survival in solid pancreas transplantation: a systematic review
}

\author{
Jonathan E. H. Ling ${ }^{1,2^{*}}$, Timothy Coughlan ${ }^{3}$, Kevan R. Polkinghorne ${ }^{1,2,4}$ and John Kanellis ${ }^{1,2}$
}

\begin{abstract}
Background: Risk indices such as the pancreas donor risk index (PDRI) and pre-procurement pancreas allocation suitability score (P-PASS) are utilised in solid pancreas transplantation however no review has compared all derived and validated indices in this field. We systematically reviewed all risk indices in solid pancreas transplantation to compare their predictive ability for transplant outcomes.

Methods: Medline Plus, Embase and the Cochrane Library were searched for studies deriving and externally validating risk indices in solid pancreas transplantation for the outcomes of pancreas and patient survival and donor pancreas acceptance for transplantation. Results were analysed descriptively due to limited reporting of discrimination and calibration metrics required to assess model performance.

Results: From 25 included studies, discrimination and calibration metrics were only reported in $88 \%$ and $38 \%$ of derivation studies $(n=8)$ and in $25 \%$ and $25 \%$ of external validation studies $(n=12)$ respectively. 21 risk indices were derived with mild to moderate ability to predict risk (C-statistics $0.52-0.78$ ). Donor age, donor body mass index (BMI) and donor gender were the commonest covariates within derived risk indices. Only PDRI and P-PASS were subsequently externally validated, with variable association with post-transplant outcomes. P-PASS was not associated with pancreas graft survival.

Conclusion: Most of the risk indices derived for use in solid pancreas transplantation were not externally validated (90\%). PDRI and P-PASS are the only risk indices externally validated for solid pancreas transplantation, and when validated without reclassification measures, are associated with 1-year pancreas graft survival and donor pancreas acceptance respectively. Future risk indices incorporating recipient and other covariates alongside donor risk factors may have improved predictive ability for solid pancreas transplant outcomes.
\end{abstract}

Keywords: Risk index, Pancreas, Transplantation, Survival, Organ acceptance, Risk

\section{Background}

Risk indices are used to predict risk and guide decisionmaking during various types of organ transplantation [1-5]. Comprising a combination of donor, recipient and transplant-related factors, risk indices are used to

\footnotetext{
*Correspondence: jlingeh@gmail.com

${ }^{1}$ Department of Nephrology, Monash Medical Centre, Monash Health,

246 Clayton Road, Clayton, Melbourne, VIC 3168, Australia

Full list of author information is available at the end of the article
}

prioritise patients on the transplant waiting list for transplantation [1] as well as to guide donor organ acceptance for transplantation $[4,6]$. Risk indices currently in use for solid pancreas transplantation are the pre-procurement pancreas allocation suitability score (P-PASS) and the pancreas donor risk index (PDRI), both comprised primarily of donor factors [3-5]. The P-PASS is currently used by countries within the Eurotransplant network to guide donor pancreas acceptance [6] while PDRI predicts pancreas graft survival and is reported in pancreas

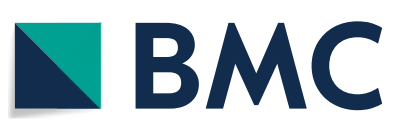

(c) The Author(s) 2021. Open Access This article is licensed under a Creative Commons Attribution 4.0 International License, which permits use, sharing, adaptation, distribution and reproduction in any medium or format, as long as you give appropriate credit to the original author(s) and the source, provide a link to the Creative Commons licence, and indicate if changes were made. The images or other third party material in this article are included in the article's Creative Commons licence, unless indicated otherwise in a credit line to the material. If material is not included in the article's Creative Commons licence and your intended use is not permitted by statutory regulation or exceeds the permitted use, you will need to obtain permission directly from the copyright holder. To view a copy of this licence, visit http://creativecommons.org/licenses/by/4.0/. The Creative Commons Public Domain Dedication waiver (http://creativeco $\mathrm{mmons}$.org/publicdomain/zero/1.0/) applies to the data made available in this article, unless otherwise stated in a credit line to the data. 
transplantation within the USA [7]. However, they are not widely used as external validation studies have reported varying association with their intended outcomes [8-11]. Other risk indices have been derived for use in pancreas transplantation however have not been validated widely in external cohorts $[12,13]$.

We compared the predictive ability of all current risk indices derived for use in solid pancreas transplantation via a systematic review. This would guide future work in incorporating a risk index into the Australian and New Zealand pancreas transplant protocol, as no index is currently used to guide solid pancreas transplantation locally $[5,14]$.

\section{Methods}

This systematic review was guided by the Cochrane's Critical Appraisal and Data Extraction for Systematic Reviews of Prediction Modelling Studies (CHARMS) tool for reviews of prediction modelling studies [15] and used the Transparent Reporting of a multivariable prediction model for Individual Prognosis or Diagnosis (TRIPOD) checklist to assess the completeness of individual study reporting [16]. This project was exempt from requiring local ethics board approval as only previously published data (no identifiable individual data) was the subject of review. The review protocol was registered via the International Prospective Register of Systematic Reviews (PROSPERO ID CRD42018080189) [17].

\section{Literature search}

Ovid Medline, Embase and the Cochrane Database of Systematic Reviews were searched for studies which derived or validated risk indices used in pancreas transplantation from inception to the $30^{\text {th }}$ of March 2020 . Grey literature searching of OpenGrey, Scopus and Web of Science was performed for the same period. Search terms included the following keywords and MESH terms; pancreas, transplant, donor, recipient, index or indices, model or models, tool or tools, pancreas after kidney (PAK), pancreas transplant alone (PTA), simultaneous kidney-pancreas transplant (SPK), P-PASS and PDRI. (Search protocols in Additional file 1: Supplement 1).

\section{Eligibility criteria}

All observational studies which derived or validated risk indices for solid pancreas transplantation were accepted for full-text review. Islet transplant studies were excluded. A risk index or model was defined as a combination of multiple predictors which calculated individual patient risk of a future outcome [18]. Studies examining a single risk factor's association with solid pancreas transplant outcomes were excluded. Likewise, case series and studies identifying factors associated with solid pancreas transplant outcomes without deriving a risk index or validating a known risk index were excluded. Studies whose aims did not include either deriving or validating a risk index but analysed PDRI or P-PASS for association with various pancreas transplant outcomes were retained for discussion but not included in the analysis. We anticipated a limited number of relevant studies and therefore included abstracts meeting inclusion criteria where no full-text article was available.

\section{Study outcomes}

Primary outcomes were pancreas graft survival, patient survival and donor pancreas acceptance for solid-organ pancreas transplantation. Pancreatic graft failure was defined as a permanent return to insulin therapy or pancreatectomy [14] and was reported as death-censored where possible based on study reporting.

\section{Data extraction and critical appraisal}

The CHARMS tool was utilized for data extraction (as data was non-randomised) [15]. Domains within CHARMS include data source, participant description, predicted outcomes, significant predictors, sample size, data handling, model development, model performance, model evaluation and results. The TRIPOD checklist was used to assess the quality of data reporting for all included studies [16]. The Prediction Model Risk of Bias Assessment Tool (PROBAST) was used to assess risk of bias and applicability of included studies [19, 20].

Model performance was assessed via study reporting of discrimination and calibration metrics [21], as described in TRIPOD, PROBAST and elsewhere [16, 20, 22, 23]. Discrimination (measured via the $\mathrm{C}$-statistic or the area under the receiver operating characteristic (AUROC) curve $[22,23])$ is the ability to distinguish those at higher risk (for outcome of choice) from those at lower risk. For instance, a C-statistic of 1.0 indicates an index is able to perfectly predict subjects at higher (or lower) risk, whereas 0.5 represents inability to differentiate between risk outcomes (akin to flipping a coin) [23]. The accuracy of an indices' predicted risk (compared to the actual absolute risk) is measured by calibration [22, 23]. This is performed via the Hosmer-Lemeshow test, comparison of calibration plots or observed-predicted outcome ratios [22-24]. A well-calibrated model is denoted by the lack of significant differences between observed and predicted outcomes or a Hosmer-Lemeshow p value of $>0.05$ [24, 25].

Model predictors, effect estimates (hazard ratios), missing data, events-per-variable (EPV) rate were extracted to assess study quality $[15,26]$. Risk indices derived in a cohort with an EPV $<10$ have a risk of overfitting (small number of outcome events compared to number of 
model predictors) $[22,23]$. When indices were externally validated, we noted if reclassification of predictors took place [27]. If a single study derived more than one risk index, they were considered unique models if the predictors within the models were different.

Two authors (JEHL and TC) performed title, abstract and full-text reviews independently and compared results. Where there was no consensus between both authors, a third author (JK or KRP) was involved. Data extraction and risk of bias assessment was performed by two authors (JEHL and TC) independently and results were compared. Study authors were contacted for clarification and data extraction (9 study authors contacted) particularly when only abstract-level data was available, but only one response was forthcoming.

\section{Data analysis}

All risk indices derived for use in solid pancreas transplantation were grouped by the outcomes they were derived to predict. If more than two studies derived risk indices for similar outcomes, we intended to meta-analyse their metrics of model performance. Unfortunately, insufficient metrics of discrimination and calibration were reported by studies deriving and externally validating risk indices to allow pooling of these metrics in a meta-analysis.

Results are therefore presented in two analyses. Firstly, we describe all risk indices derived to predict our primary outcomes in solid pancreas transplantation. Secondly, we describe the external validation of these risk indices. For each analysis, we report risk index performance via their discrimination and calibration metrics (where present) and assess their method of derivation, association with outcome, study quality and risk of bias. We used the Grading of Recommendations, Assessment, Development and Evaluations (GRADE) framework to summarize the current evidence for the use of externally validated risk indices by outcome, according to the domains of Risk of bias, Inconsistency, Indirectness, Imprecision and Publication bias [28-30].

\section{Results}

5715 abstracts were identified. After deduplication, 5554 studies underwent title/abstract screening and 66 studies proceeded to full-text screening. After further exclusions, 25 studies were included in the review. The PRISMA (Preferred Reporting Items for Systematic Reviews and Meta-Analyses) flow diagram [31] is included in Fig. 1. Eight studies derived 21 risk indices predicting our primary outcomes (including PDRI and P-PASS) [3, 4, 11-13, 32-35] (Table 1). From these derived risk indices, only PDRI and P-PASS (Table 2) were externally validated in 19 studies. Regarding TRIPOD assessment of completeness of data recording for all studies, only 20 of all 33 domains recorded $>70 \%$ adherence (Additional file 1: Supplement 2).

\section{Studies deriving risk indices: model performance}

All eight studies deriving risk indices were retrospective. Five studies derived one risk index each $[3,4,11$, $12,32]$, two studies derived two risk indices each [13, 35 ] and one study derived 12 risk indices [33] for outcomes post-solid pancreas transplant (Table 1). In all, 13 donor predictors, 14 recipient predictors and 10 other predictors were used to derive 21 risk indices (Fig. 2a-c). The commonest predictor was donor age $(n=8)$, followed by donor body mass index (BMI) $(n=6)$ and donor gender $(n=4)$.

Discrimination metrics (AUROC/C-statistic) were reported by seven studies (88\%) deriving indices (Table 1). Three risk indices predicting 3-month pancreas survival reported C-statistics of $0.52-0.78[12,13$, 35]. In comparison, five indices (including PDRI) predicting 1-year pancreas survival reported C-statistics of $0.61-0.78[3,11,33]$. Five risk indices for 1-year patient survival reported C-statistics of $0.62-0.8$ [32, 33, 35]. Six risk indices reported C-statistics of 0.59 to 0.66 for 3 -year pancreas survival and 0.64 to 0.76 for 3 -year patient survival [33]. The study deriving P-PASS did not report model discrimination for donor pancreas acceptance [4]. Overall, minimal to moderate ability to predict risk was present (where reported) for the studies reporting discrimination metrics.

Model calibration (Hosmer-Lemeshow test or observed/predicted events ratio) was reported by three studies (38\%) deriving indices (Table 1). The logistic regression model by Dorsey et al. [13] had a C-statistic of 0.78 for 3-month pancreas survival, with a HosmerLemeshow p value of 0.74 . In comparison, the Composite Risk Model [12] had C-statistics of 0.6 to 0.52 for the same outcome depending on the number of risk factors included. The corresponding observed/predicted ratios decreased from 0.8 to 0.2 (with increasing risk factors), with a concurrent decrease in model sensitivity. Meanwhile Kasiske et al. derived 12 models with $\mathrm{C}$-statistics ranging from 0.61 to 0.78 for 1 - and 3-year pancreas and graft survival (by transplant type) and reported Hosmer-Lemeshow p values ranging from 0.24 to 0.92 [33]. The PDRI C-statistic was 0.67 for 1 -year pancreas survival with no calibration reported [3]. No discrimination was reported for P-PASS however the observed incidence of declining a donor pancreas $(45.3 \%)$ corresponded to the predicted risk of declining a donor pancreas $(42.8 \%)$ with a P-PASS $\geq 17$ [4]. 
PRISMA Flow Diagram

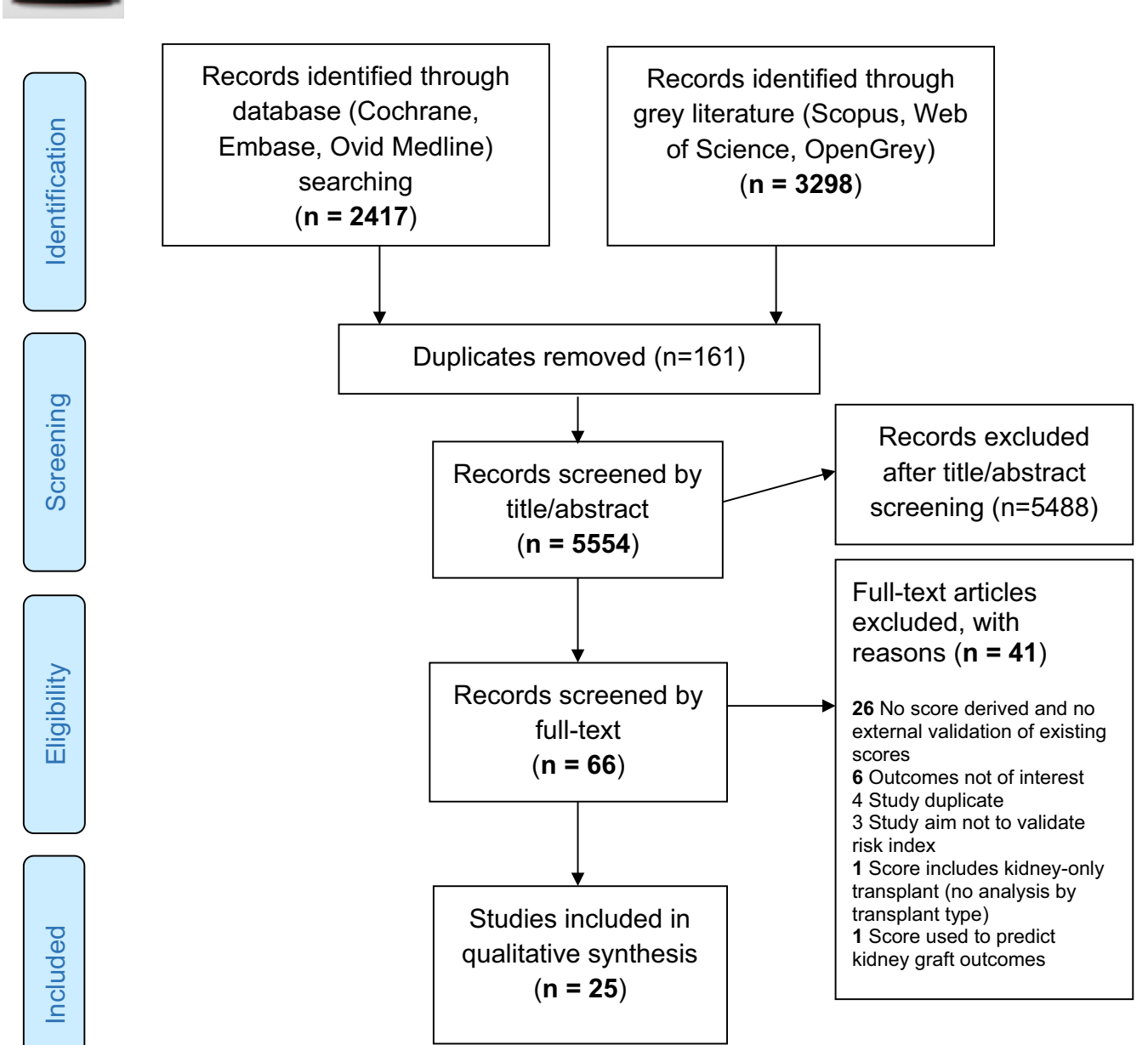

Fig. 1 PRISMA flow diagram

Studies deriving risk indices: study quality and risk of bias Overall study quality of studies deriving risk indices was moderate (Table 1, Additional file 1: Supplement 3 ). Four studies were in single centre cohorts, three studies used registry data, and one study utilized a multi-centre cohort. Cohorts consisted of recipients of all solid pancreas transplant types (SPK, PAK, PTA) in seven of eight studies deriving risk indices (one study was in an SPK-only cohort [32]). Study outcomes (graft survival) were defined in seven of eight studies, with centre-based reporting of graft survival by one registry-based study [3] (Additional file 1: Supplement 3). Outcomes reported were 3-month pancreas survival $(\mathrm{n}=3), 1$-year pancreas survival $(\mathrm{n}=3), 1$-year patient survival $(n=2), 3$-year pancreas and patient survival $(\mathrm{n}=1)$ and donor pancreas acceptance $(\mathrm{n}=1)$ (Table 1$)$. Five of eight studies had an events-per-variable (EPV) rate of $>10$, lowering the risk of overfitting (Additional file 1: Supplement 3). Missing data was present in one study [11] and was handled via complete case analysis. In three studies, it was unclear whether missing data was present [32, 33, 35]. Four studies had no missing data $[3,4,12,13]$ (Additional file 1: Supplement 3).

Risk indices were modeled differently between studies. The PDRI was developed from significant donor predictors identified via multivariate Cox regression and combined into a continuous risk index with the median donor having a PDRI of 1.0 [3]. In comparison, P-PASS used pre-defined predictors identified by expert opinion to derive logistic regression models for 


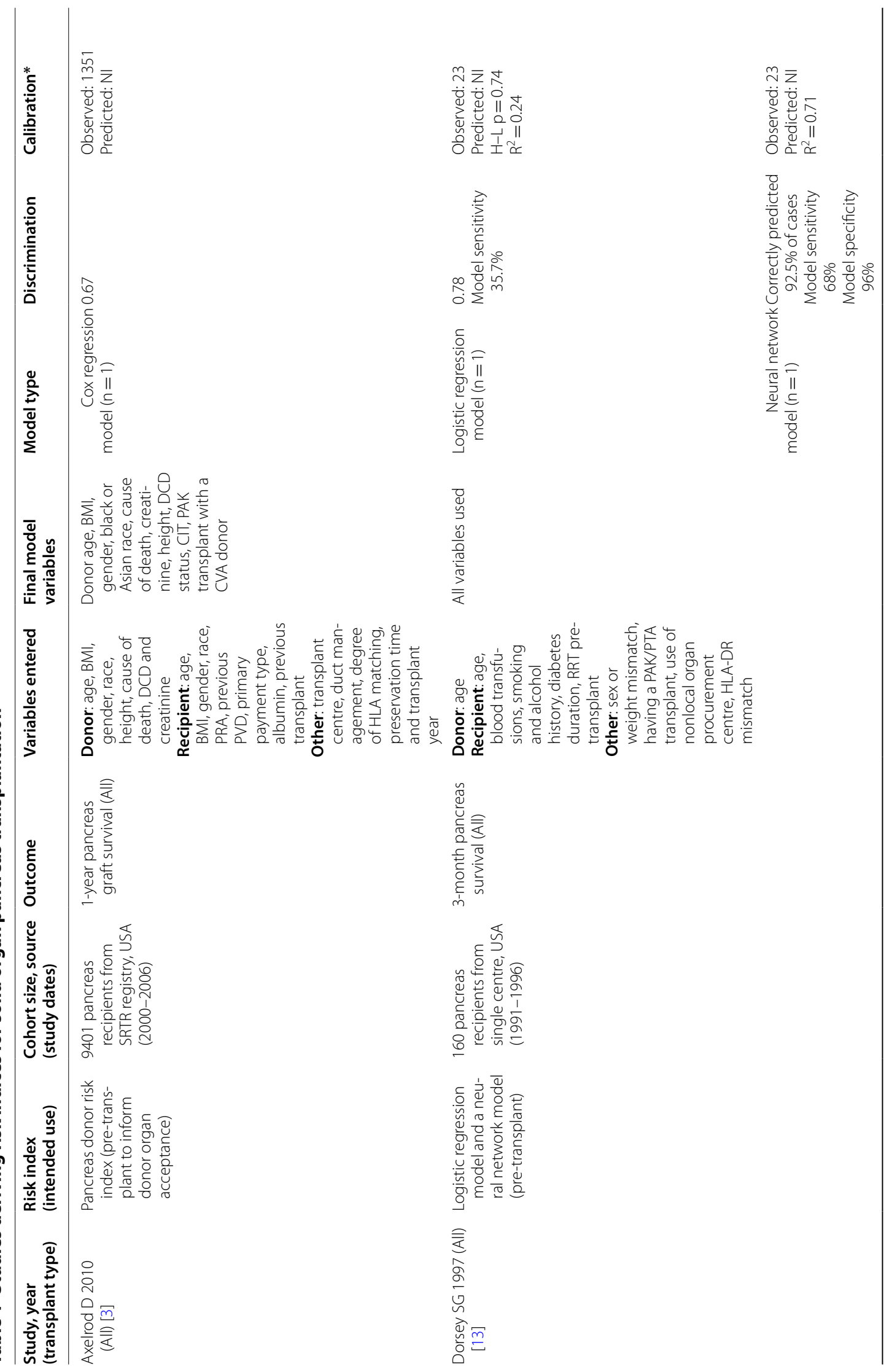




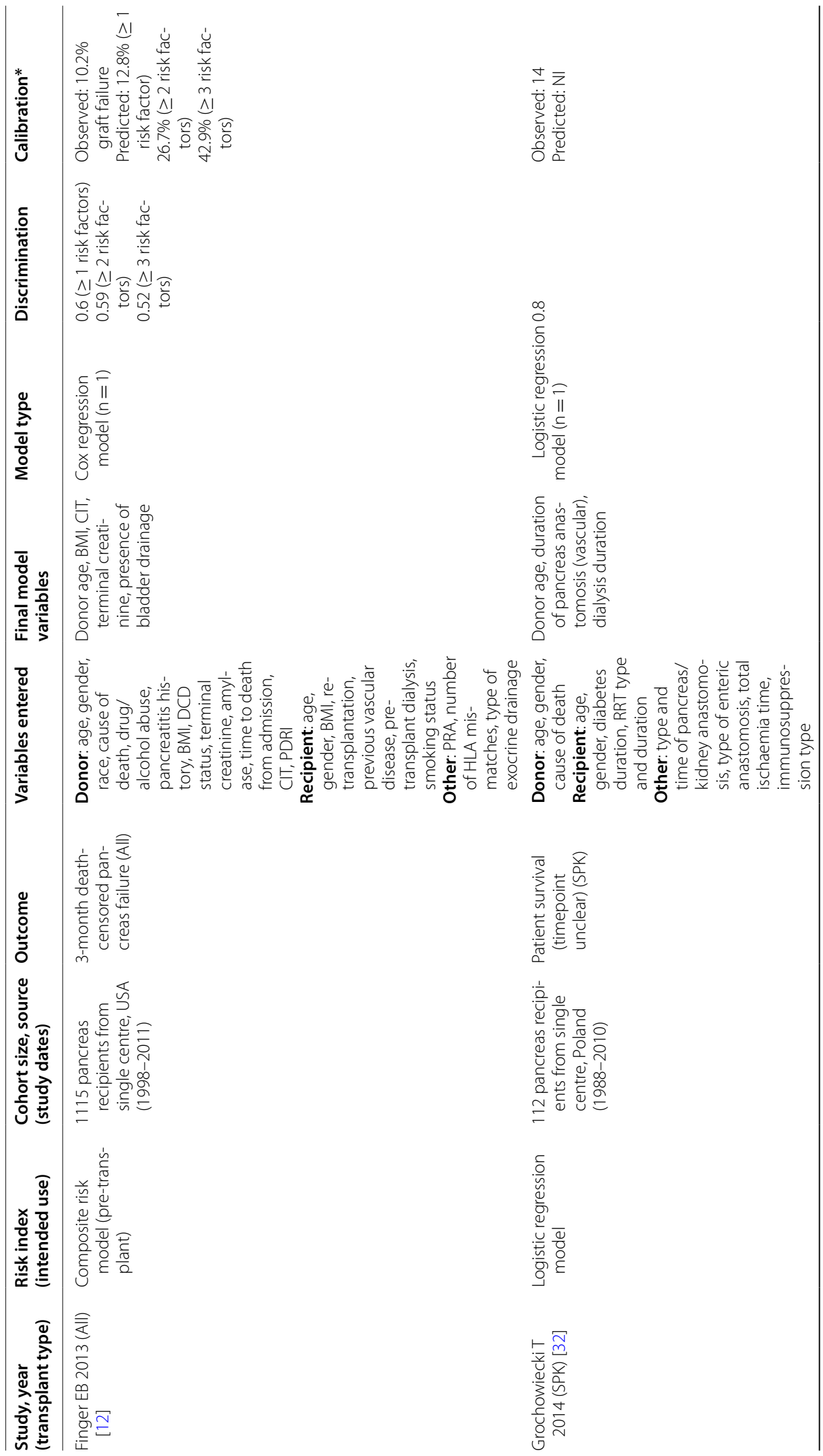




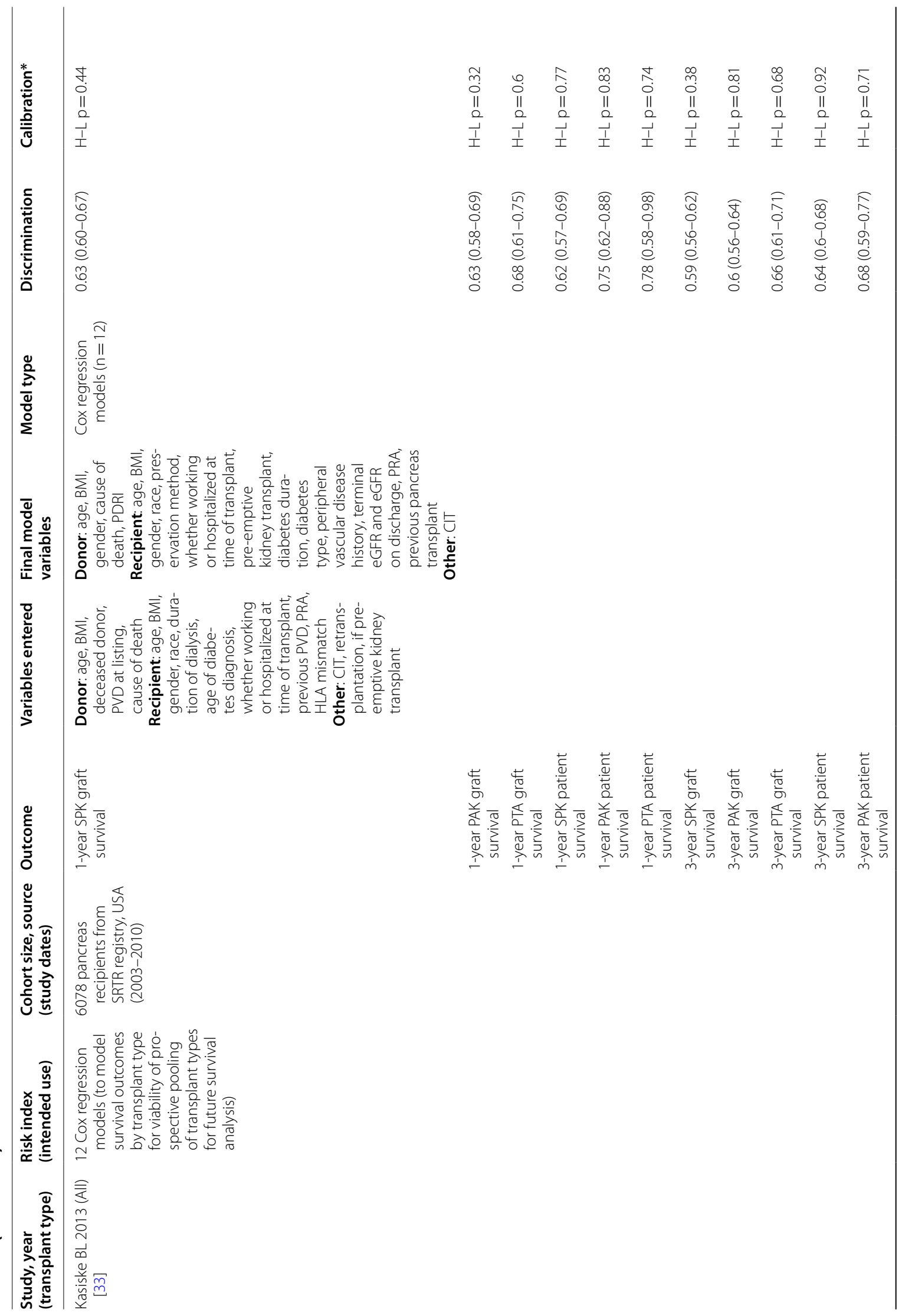




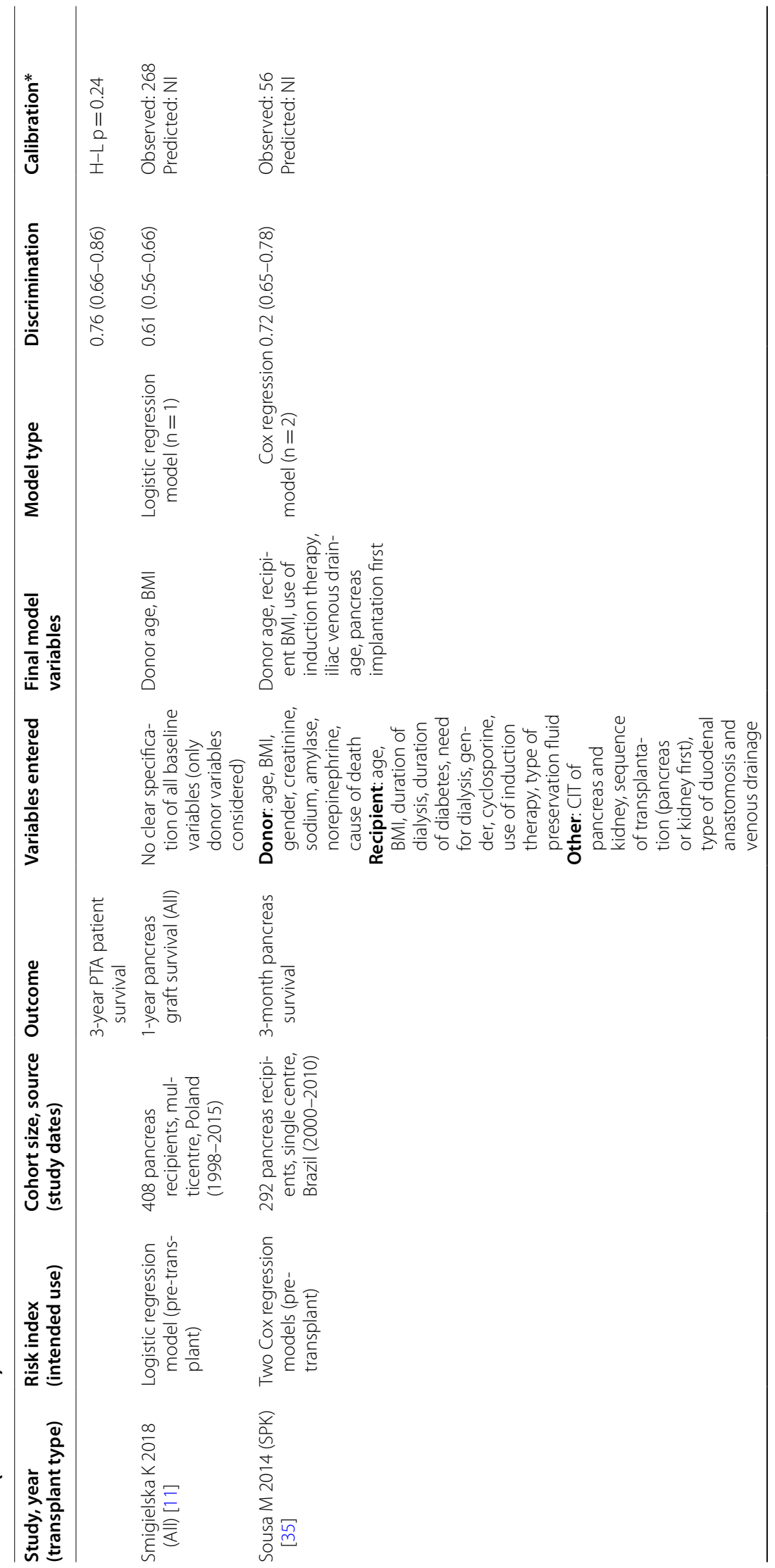




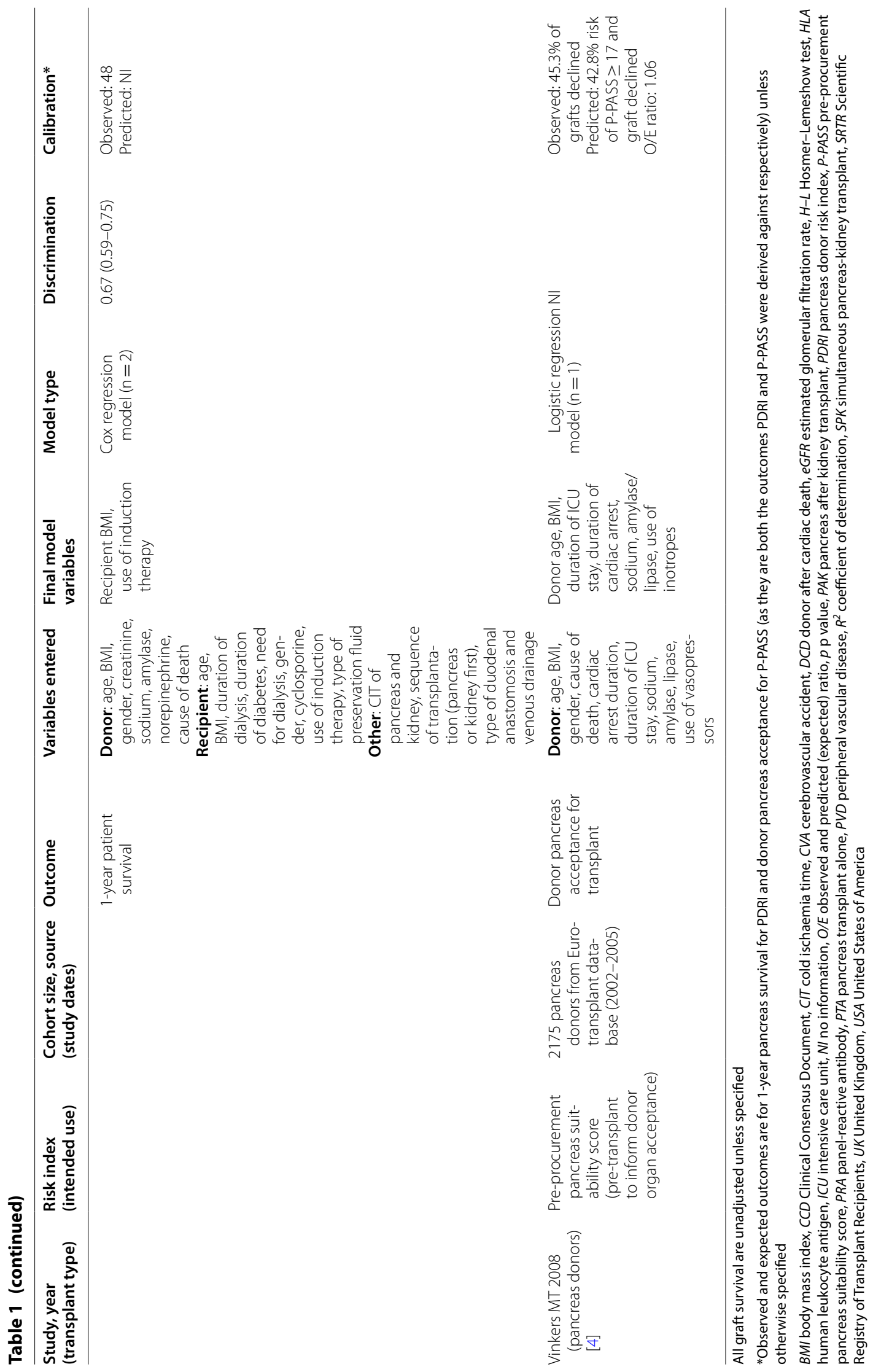


Table 2 PDRI and P-PASS predictors

\begin{tabular}{ll}
\hline $\begin{array}{l}\text { P-PASS (Pre-procurement } \\
\text { pancreas suitability score) }\end{array}$ & PDRI (Pancreas donor risk index) \\
\hline Age & Age \\
BMI & BMI \\
ICU stay (days) & Gender \\
Cardiac arrest (min) & Asian race \\
Serum sodium (mmol/L) & Black race \\
Amylase (U/L) or & Height (cm) \\
Lipase (U/L) & Cause of death (if CVA/stroke) \\
(Nor)adrenaline or & CVA/stroke in PAK \\
Dobuta-/dopamine & Cold ischaemia (h) \\
& DCD status \\
& Serum creatinine (if $>2.5$ mg/dl) \\
Donor P-PASS $>173$ times more & Increased PDRI associated with \\
likely to be refused & reduced 1-year pancreas graft \\
& survival
\end{tabular}

BMI body mass index, CIT cold ischaemia time, CVA cerebrovascular accident, $D C D$ donor after cardiac death, ICU intensive care unit, PAK pancreas after kidney transplant, $P D R /$ pancreas donor risk index, $P$-PASS pre-procurement pancreas suitability score

the binary outcomes of donor pancreas acceptance [4]. Initially derived as a continuous score, P-PASS was reclassified into equally-sized categories which were gradually collapsed when regression coefficients were similar, culminating in a model with two categories. Similarly, Dorsey et al. utilized predictors from medical expertise to derive a logistic regression model predicting 3-month pancreas survival. A second model was derived using a backpropagation neural network with all predictors entered as input nodes [13]. Risk indices from other studies were derived using the regression coefficients of significant predictors from multivariate analysis [11, 12, 32, 33, 35]. Only one study (out of eight) derived risk indices by each pancreas transplant type [33]. Only four studies (50\%) reported derived risk index equations containing all final predictors with coefficients $[3,11,12,32]$ and only four studies (50\%) documented internal validation procedures (Additional file 1: Supplement 3) [3, 4, 12, 33].

The overall PROBAST for studies deriving risk indices was rated at high risk of bias and low applicability (Table 3). All studies except one [13] were at high risk of bias for the 'Analysis' domain due to limited reporting of discrimination and calibration metrics. The other PROBAST domains for 'Participants', 'Predictors' and 'Outcomes' had low risk of bias in eight (100\%), four (50\%) and seven (88\%) studies respectively. Four studies (50\%) scored poorly for 'Applicability' as they included factors that could only be measured at the transplant stage (such as cold ischaemia) or post-transplant stage (such as iliac venous drainage or use of induction therapy), despite being derived for use pre-transplant $[12,32,33,35]$.

\section{Studies externally validating risk indices: model performance}

Of the derived risk indices, only P-PASS and PDRI were further validated in 18 studies (PDRI in 11 studies [8, 9, $11,12,36-42]$ and P-PASS in 14 studies $[8,10,11,34$, 37-40, 43-47] respectively). However, of these studies, PDRI and P-PASS were validated against their outcomes that they were derived to predict only in 12 studies (PDRI in nine studies $[8,9,11,36-39,42]$ and P-PASS in three studies [34, 40,45] respectively) (Table 4). These studies proceeded to undergo study quality and risk of bias assessment. Studies examining PDRI and P-PASS against outcomes for which they were not derived to predict are listed in Additional file 1: Supplement 4.

Only two of the 12 studies (17\%) externally validating PDRI reported discrimination metrics. Blok et al. reported a C-statistic of 0.69 for PDRI for an association between PDRI and pancreas survival up to 10 years when a cut-off of 1.24 was used [8]. This was similar to the C-statistic reported in the PDRI derivation study. Smigielska et al. reported a AUROC of 0.52 for PDRI as a continuous model in predicting 1-year pancreas survival but found that PDRI was not associated with the outcome [11]. Only one of 12 studies (8\%) validating PDRI reported observed/expected ratios for calibration (ranging from 0.76 to 1.12 by quintile and transplant type) [41] however the study deriving PDRI [3] did not report calibration metrics hence no comparison was possible.

Of the four studies utilizing PDRI by quintiles (as per its' derivation), two reported an association between PDRI and pancreas survival (only in SPK transplants from two studies) [41, 42]. Of the three studies utilizing PDRI as a continuous model, one study reported an association between PDRI and 1-year pancreas survival [42]. From the five studies validating PDRI via different risk groups to derivation, only one study reported an association with pancreas survival [8] suggesting that reclassification of PDRI during external validation may have affected the outcome.

For P-PASS, one of three (33\%) external validation studies reported discrimination and calibration metrics. Kopp et al. reported a C-statistic of 0.68 for P-PASS and the outcome of donor pancreas acceptance [40]. However, observed/exposed ratios of 0.69 to 0.72 were reported by two studies $(67 \%)[34,40]$ compared to P-PASS derivation (observed/exposed ratio 1.06) [4]. All three studies validating P-PASS for donor pancreas acceptance reported an association with the outcome [34, $40,45]$. 


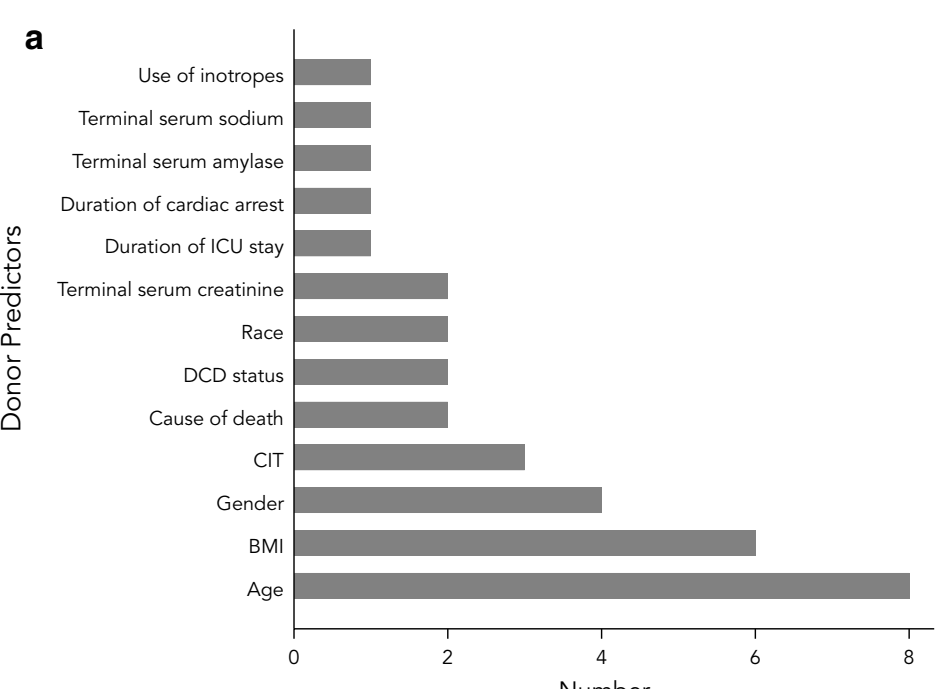

b
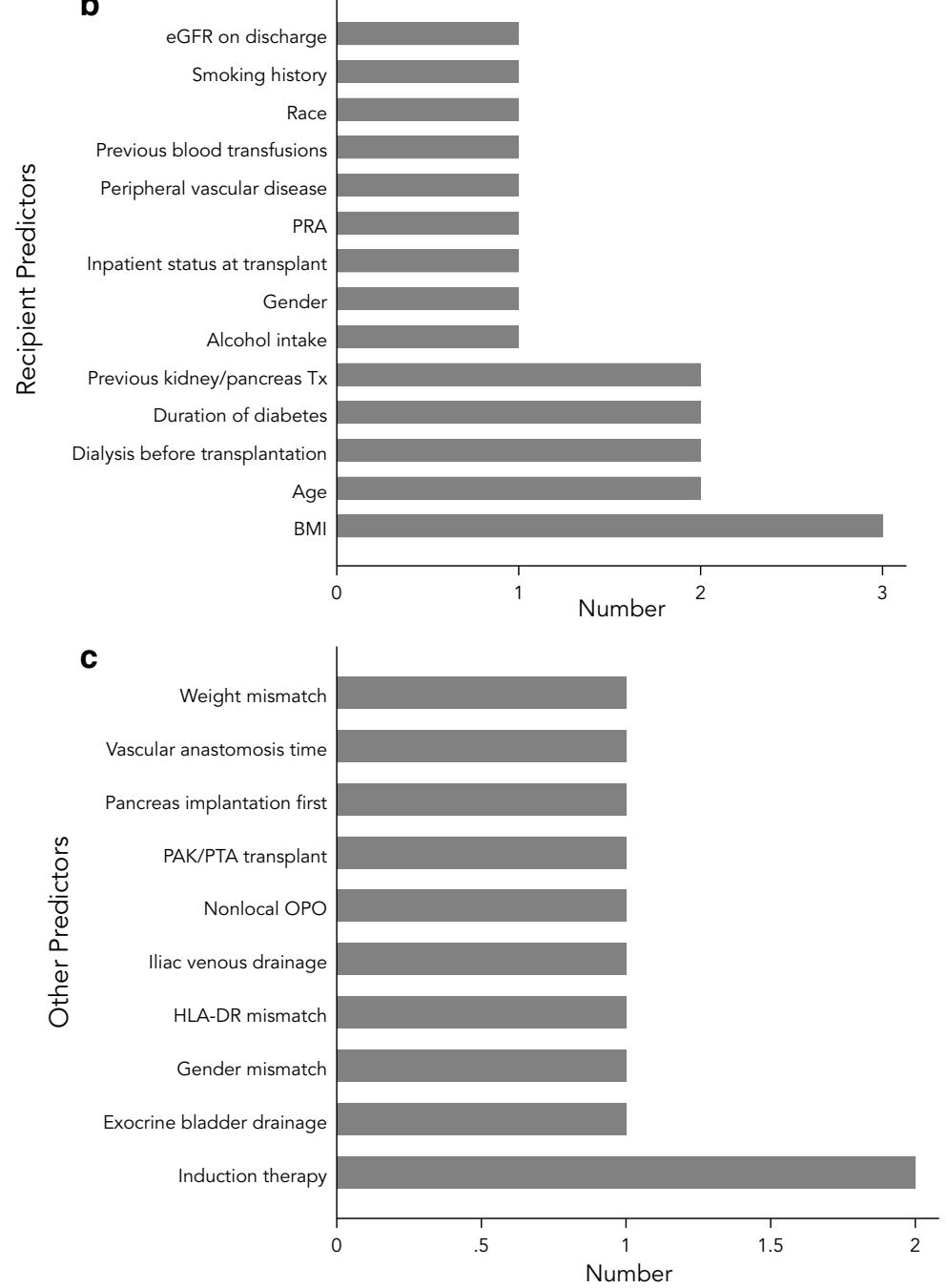

Fig. 2 Distribution of donor predictors (a), recipient predictors (b) and other predictors (c) within risk indices derived for use in solid pancreas transplantation 
Table 3 PROBAST assessment for studies deriving risk indices

\begin{tabular}{|c|c|c|c|c|c|c|c|c|c|}
\hline \multirow[t]{2}{*}{ Study } & \multicolumn{4}{|l|}{ Risk of Bias } & \multicolumn{3}{|l|}{ Applicability } & \multicolumn{2}{|l|}{ Overall } \\
\hline & Partcipants & Predictors & Outcome & Analysis & Participants & Predictors & Outcome & Risk of bias & Applicability \\
\hline Axelrod DA 2010 [3] & + & + & - & - & + & - & - & - & + \\
\hline Dorsey SG 1997 [13] & + & + & + & + & + & + & + & + & + \\
\hline Finger EB 2013 [12] & + & - & + & - & + & - & + & - & - \\
\hline Grochowiecki T 2014 [32] & + & - & + & - & + & - & - & - & - \\
\hline Kasiske BL 2013 [33] & + & - & + & + & + & - & + & - & - \\
\hline Smigielska 2018 [11] & + & + & + & - & + & + & + & - & + \\
\hline Sousa M 2014 [35] & + & - & + & - & + & - & + & - & - \\
\hline Vinkers MT 2008 [4] & + & + & + & - & + & + & + & - & + \\
\hline
\end{tabular}

PROBAST Prediction model Risk of Bias ASsesment Tool, $R O B$ risk of bias

+ indicates low ROB/low concern regarding applicability; - indicates high ROB/high concern regarding applicability; ? indicates unclear ROB/unclear concern regarding applicability

\section{Studies externally validating risk indices: study quality and risk of bias}

Overall study quality of the external validation studies was poor (Table 4). Of the 12 external validation studies, eight studies utilized single-centre cohorts, two studies were registry-based, and one study utilized a multi-centre cohort. Missing data was present in eight studies (62\%), varying from 1.4 to $73 \%$ of the cohort [8, $9,11,36,37,39,40,42]$. This was handled by complete case analysis in all eight studies. Three studies had no missing data $[34,38,41]$ and one study did not report missing data [45]. Graft failure was not defined in three studies (23\%) [36, 39, 45]. Model predictors were reclassified in several studies. In five studies, PDRI was categorised differently to how it was originally derived [8, 36-39]. In these studies, PDRI was classified either as 'high' or 'low' according to the median PDRI within the cohort, or in tertiles. Furthermore, donor race was classified differently to that of PDRI derivation in one study due to differences in that country [36] and was not clearly classified in two studies [11, 38] (not reported with other study variables). P-PASS was also validated while omitting serum sodium in one study due to lack of reporting in that jurisdiction [45].

Due to the limited reporting of discrimination and calibration metrics, risk of bias for the 'Analysis' domain in PROBAST was high in all but one study [40] (Table 5). However, domains for 'Participants', 'Predictors' recorded low risk of bias for all 12 studies, while 'Outcomes' had low risk of bias in nine studies (75\%). 'Applicability' was rated low for two studies(17\%) [36, 45] due to predictors being modified as previously described, and unclear for four studies [11, 38, 39, 41] due to lack of information on outcome definition and predictor collection.

\section{GRADE assessment}

All derivation and external validation studies were included in the GRADE assessment of the overall quality of evidence by outcomes. Baseline evidence quality was downgraded to 'Moderate' as all studies were retrospective and non-randomised [30]. This was further downgraded to 'Low' due to the high risk of bias as per PROBAST. For studies examining PDRI as a continuous score as well as via various risk categories, GRADE was downgraded for 'Inconsistency' domain as varying degrees of association with the outcome were present. For studies validating PDRI using different PDRI risk groups (to its' derivation) GRADE was downgraded for the 'Indirectness' domain. Some outcomes included only one or two studies or were performed in small cohorts with low event rates, thus GRADE was downgraded for 'Imprecision' (Table 6).

In summary, 'Low' quality evidence exists for PDRI (as quintiles per derivation) in predicting risk of 1-year pancreas survival and for P-PASS in predicting donor pancreas acceptance (Table 6). Even less evidence exists in utilizing PDRI by different risk strata to its' derivation, and for PDRI in predicting 1-year patient survival.

\section{Discussion}

This systematic review of risk indices derived for use in solid pancreas transplantation found that despite 21 risk indices being derived, only P-PASS and PDRI were externally validated and are in use today [6, 7]. PDRI (derived in USA) was validated in a UK cohort $[41,42]$ while P-PASS (derived in the Netherlands) was validated in Spanish [34] and Australian [45] cohorts, albeit in a modified form in the latter.

PDRI discrimination for 1-year pancreas survival was poor to moderate (C-statistic/AUROC 0.52-0.69) and 


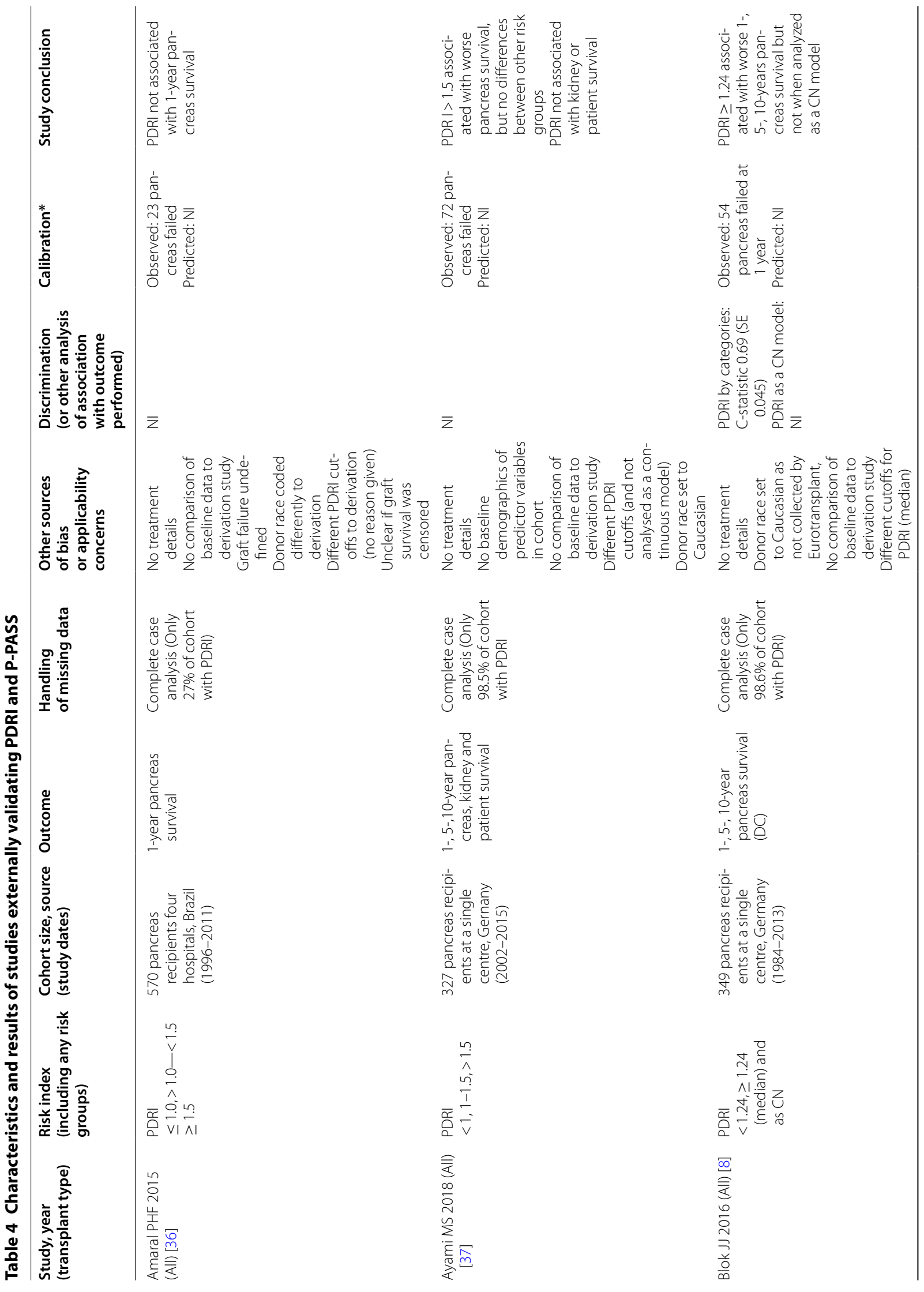




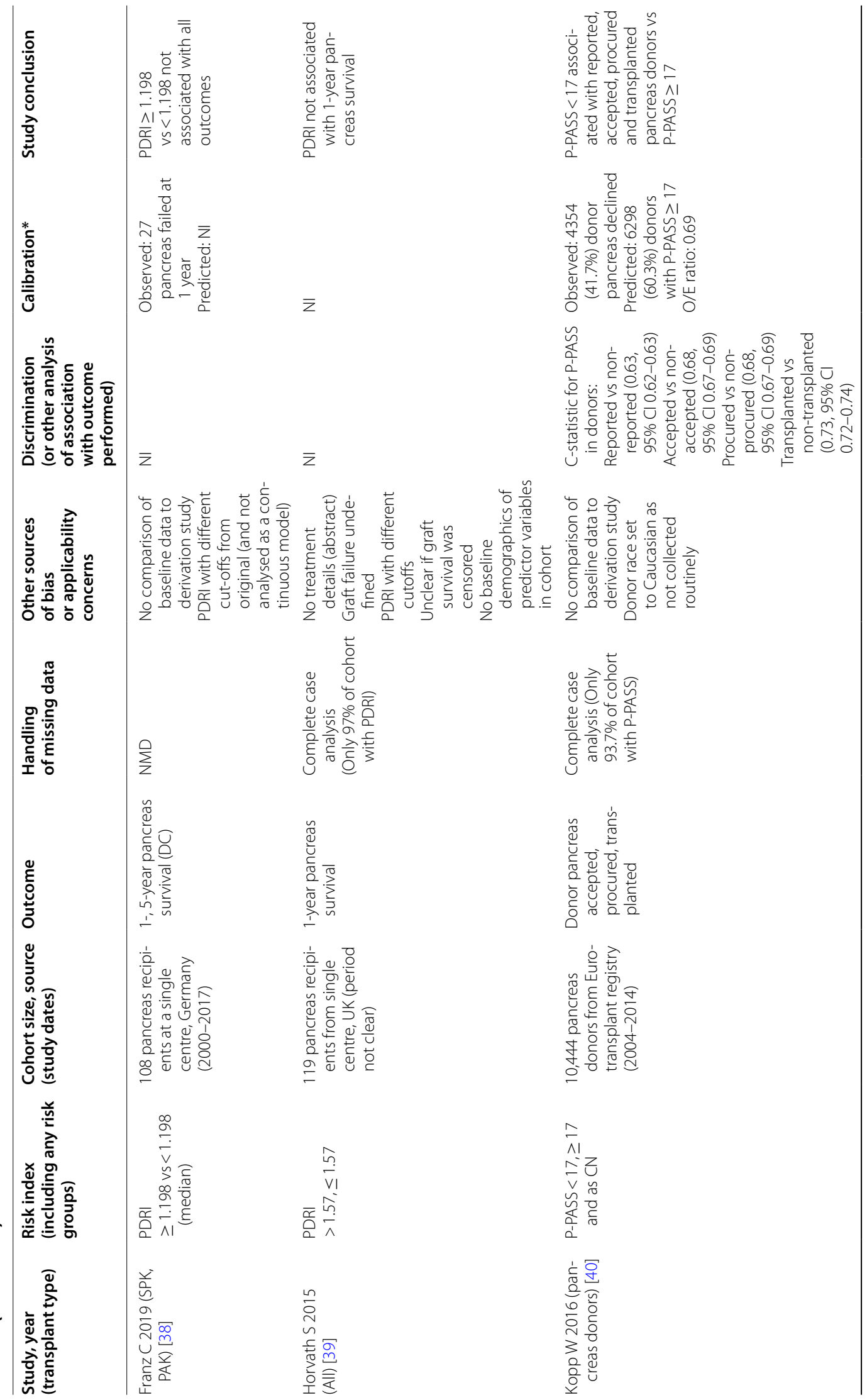




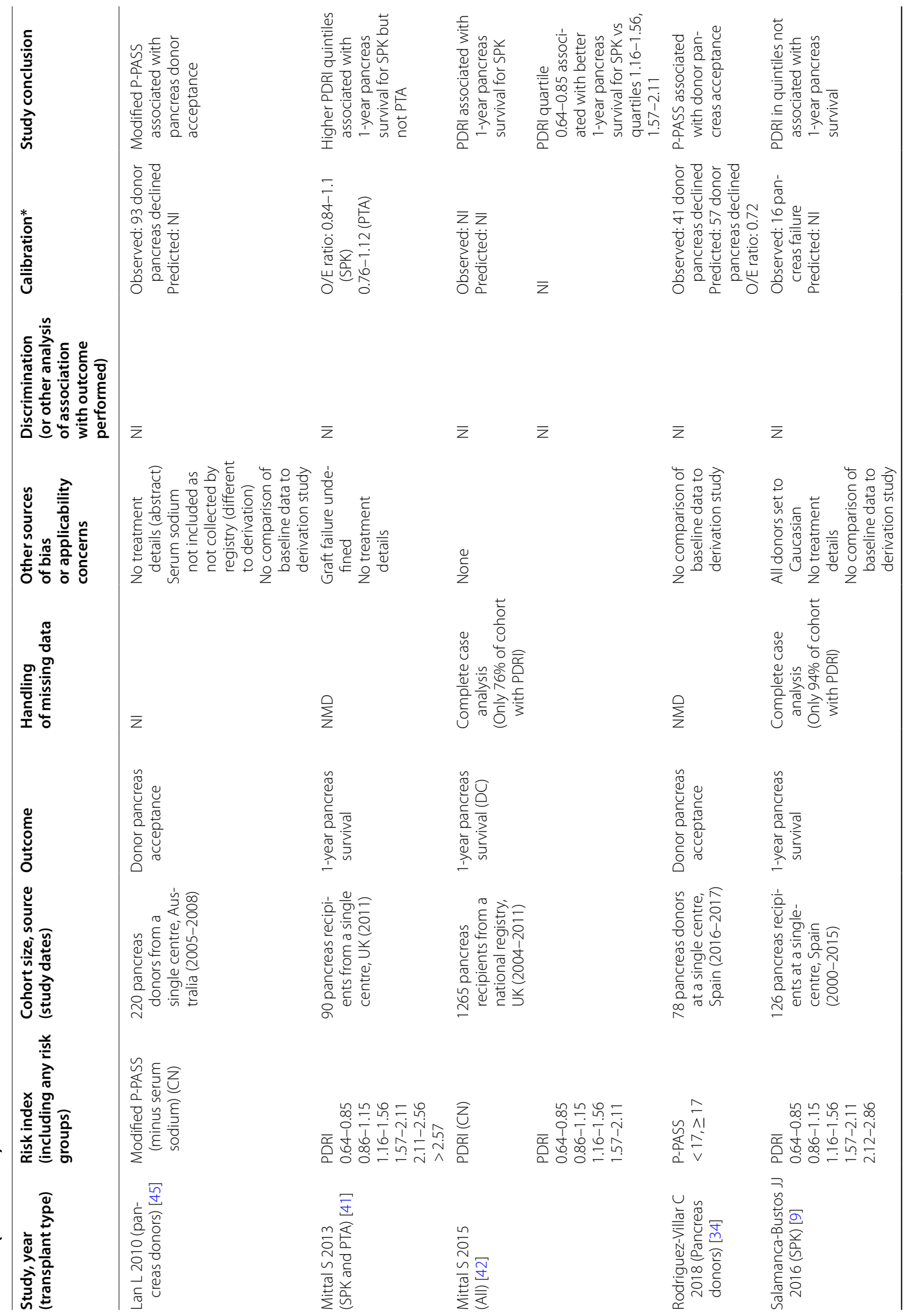


P-PASS discrimination was moderate for donor pancreas acceptance (C-statistic 0.68). Calibration was poorly reported in both PDRI and P-PASS derivation and external validation studies. In P-PASS external validation studies, calibration (in form of $\mathrm{O} / \mathrm{E}$ ratios) was lower than in its' derivation, suggesting a degree of overestimation. A contributing factor to this from one study was that donor pancreas acceptance in that particular cohort was determined by more liberal donor pancreas acceptance cutoffs compared to the P- PASS derivation study [34].

Within the studies included in our review, discrimination and calibration metrics were only reported in $88 \%$ and $38 \%$ of risk index derivation studies, $17 \%$ and $8 \%$ of studies externally validating PDRI and 33\% and $67 \%$ of studies externally validating P-PASS respectively. This limits the applicability of such indices in other cohorts external to its' derivation. Limited reporting of these metrics in prediction modelling has been previously reported in a systematic review of clinical prediction studies in 2008, where discrimination and calibration were only reported in $27 \%$ and $12 \%$ of studies respectively [48]. This led to the introduction of TRIPOD to ensure completeness of data reporting in prediction studies [16]. A review of studies published before TRIPOD's inception found incomplete information to guide use of prediction models was present in $>80 \%$ of derived models [49], an issue also present in our review (Additional file 1: Supplement 2).

Our review also identified 13 studies analysing the association of PDRI $(n=2)$ and P-PASS $(n=11)$ for outcomes they were not derived to predict (Additional file 1: Supplement 4). P-PASS was analysed for an association with pancreas survival in 11 studies $[8,10,11,37-39,43$, $44,46,47,50]$ (no significant association in eight studies). PDRI was associated with graft survival at 3 months in one study [12], and associated with donor pancreas acceptance in another study [40]. Therefore P-PASS in particular should not be utilised to predict pancreas survival outcomes. Our review also demonstrates that using PDRI with different risk categories to that of its' derivation, or in different cohorts without proper reclassification measures reduces its' predictive ability for pancreas survival.

Elsewhere, PDRI and P-PASS have been analysed along with other independent variables for associations with post-transplant outcomes. PDRI was associated with 1 -year pancreas and patient survival in a study examining the correlation of immunological matching with graft rejection and survival in pancreas transplantation [51]. P-PASS however was not associated with pancreas survival in a study analysing donor and recipient factors predicting graft survival post-pancreas transplantation [52], again correlating with our finding of P-PASS being 
Table 5 PROBAST risk of bias assessment for studies externally validating PDRI/P-PASS

\begin{tabular}{|c|c|c|c|c|c|c|c|c|c|}
\hline \multirow[t]{2}{*}{ Study } & \multicolumn{4}{|l|}{ Risk of bias } & \multicolumn{3}{|l|}{ Applicability } & \multicolumn{2}{|l|}{ Overall } \\
\hline & Participants & Predictors & Outcome & Analysis & Participants & Predictors & Outcome & Risk of bias & Applicability \\
\hline Amaral PHF 2015 [36] & + & + & $?$ & - & + & - & $?$ & - & - \\
\hline Ayami M 2016 [37] & + & + & + & - & + & + & + & - & + \\
\hline Blok JJ 2016 [8] & + & + & + & - & + & + & + & - & + \\
\hline Franz C 2019 [38] & + & + & + & - & + & $?$ & + & - & $?$ \\
\hline Horvath S 2015 [39] & + & + & - & - & + & $?$ & $?$ & - & - \\
\hline Kopp W 2016 [40] & + & + & + & + & + & + & + & + & + \\
\hline Lan L 2010 [45] & + & + & + & - & + & - & + & - & - \\
\hline Mittal S 2013 [41] & + & + & $?$ & - & + & + & $?$ & - & $?$ \\
\hline Mittal S 2015 [42] & + & + & + & - & + & + & + & - & + \\
\hline Rodriguez-Villar C 2018 [34] & + & + & + & - & + & + & + & - & + \\
\hline Salamanca-Bustos JJ 2016 [9] & + & + & + & - & + & + & + & - & + \\
\hline Smigielska K 2018 [11] & + & + & + & - & + & $?$ & + & - & $?$ \\
\hline
\end{tabular}

PROBAST Prediction model Risk of Bias ASsesment Tool, $R O B$ risk of bias

+ indicates low ROB/low concern regarding applicability; - indicates high ROB/high concern regarding applicability;? indicates unclear ROB/unclear concern regarding applicability

Table 6 GRADE assessment of included outcomes for PDRI and P-PASS

\begin{tabular}{|c|c|c|c|c|c|}
\hline Outcome & Risk index & Studies^ & GRADE quality & Footnotes* & Interpretation \\
\hline \multirow[t]{3}{*}{ 1-year pancreas survival } & PDRI as a continuous score & 4 & $\begin{array}{l}\oplus \\
\text { VERY LOW }\end{array}$ & $\begin{array}{l}2 \text { studies demonstrated an } \\
\text { association with outcome } \\
\text { Downgraded for: } \\
\text { Outcomes differing between } \\
\text { studies (INCONSISTENCY) }\end{array}$ & $\begin{array}{l}\text { Very low quality evidence that } \\
\text { PDRI as a continuous variable } \\
\text { is associated with 1-year } \\
\text { pancreas survival }\end{array}$ \\
\hline & PDRI as quintiles & 4 & $\begin{array}{l}\oplus \oplus \\
\mathrm{LOW}\end{array}$ & $\begin{array}{l}3 \text { of } 4 \text { studies demonstrated } \\
\text { an association with the } \\
\text { outcome } \\
\text { Association with SPK trans- } \\
\text { plants in } 2 \text { studies }\end{array}$ & $\begin{array}{l}\text { Low quality evidence that PDRI } \\
\text { in quartiles is associated with } \\
1 \text {-year pancreas survival (par- } \\
\text { ticularly SPK transplants) }\end{array}$ \\
\hline & $\begin{array}{l}\text { PDRI as categorical model } \\
\text { (various risk groups) }\end{array}$ & 5 & $\begin{array}{l}\oplus \\
\text { VERY LOW }\end{array}$ & $\begin{array}{l}1 \text { of } 5 \text { studies demonstrated } \\
\text { an association with out- } \\
\text { come } \\
\text { Downgraded for: } \\
\text { Differing PDRI risk groups } \\
\text { (INDIRECTNESS) } \\
\text { Outcomes differing between } \\
\text { studies (INCONSISTENCY) } \\
\text { Small total event rate }<300 \\
\text { (IMPRECISION) }\end{array}$ & $\begin{array}{l}\text { Very low quality evidence that } \\
\text { PDRI as a categorical model } \\
\text { (by various risk groups) is } \\
\text { associated with 1-year pan- } \\
\text { creas survival }\end{array}$ \\
\hline 1-year patient survival & PDRI as tertiles & 1 & $\begin{array}{l}\oplus \\
\text { VERY LOW }\end{array}$ & $\begin{array}{l}\text { No association with outcome } \\
\text { Downgraded for: } \\
\text { Small number of studies } \\
\quad \text { (IMPRECISION) }\end{array}$ & $\begin{array}{l}\text { Very low quality evidence that } \\
\text { PDRI by tertiles is associated } \\
\text { with 1-year patient survival }\end{array}$ \\
\hline Donor pancreas acceptance & P-PASS $<17 v S \geq 17$ & 4 & $\begin{array}{l}\oplus \oplus \\
\mathrm{LOW}\end{array}$ & $\begin{array}{l}\text { All studies demonstrated an } \\
\text { association with outcome }\end{array}$ & $\begin{array}{l}\text { Low quality evidence that } \\
\text { P-PASS is associated with } \\
\text { donor pancreas acceptance }\end{array}$ \\
\hline
\end{tabular}

*No pooling of discrimination metrics or other effect estimates unless otherwise stated. All outcome evidence was downgraded at baseline due to the observational nature of the studies as well as their risk of bias

PDRI pancreas donor risk index, P-PASS pre-procurement pancreas suitability score, SPK simultaneous pancreas-kidney transplant

poorly associated with pancreas graft survival as it was not derived to predict this.
A study limitation was the inclusion of abstracts as we anticipated a limited number of studies meeting our inclusion criteria. To counter this, we contacted 
study authors to obtain supporting information for any abstracts meeting our inclusion criteria however received few responses. Also, the use of C-statistic/ AUROC as a means of assessing discrimination has been discussed elsewhere as these metrics do not account for study heterogeneity and the predicted probabilities of individual variables upon the outcome [53]. Notwithstanding this, the TRIPOD assessment of discrimination includes the $\mathrm{C}$-statistic/AUROC hence we have considered it an acceptable metric for this purpose. Finally, as risk indices are used in combination with clinical judgement to make transplantation decisions, other factors may confound the results. Studies externally validating risk indices should compare their baseline cohort characteristics to that of the risk index derivation study, as well as other factors such as the immunosuppression regimen used in order to stratify for key differences. Unfortunately, comparisons of baseline study characteristics were not made in $75 \%$ of external validation studies and immunosuppression regimen were not detailed in $67 \%$ of external validation studies (Table 4).

Beyond the limited number of quality external validation studies, P-PASS and PDRI have other factors limiting their use in other cohorts. While able to predict which donor pancreata should be accepted, P-PASS was not derived to predict post-transplant outcomes [4] hence limiting its' ability to meaningfully guide pancreas transplantation decisions. While PDRI is associated with pancreas graft survival, it's predictive ability is best at differentiating risk between extreme PDRI values [3], thus PDRI values close to the median are less easy to interpret. Furthermore in some cohorts, PDRI has only been able to predict graft survival for SPK transplantation (as opposed to PAK or PTA transplants) [42].

Current solid pancreas transplant protocols identify suitable pancreas donors without established highrisk factors (i.e. cause of death from trauma, age below 40-50 years old, BMI under $30 \mathrm{~kg} / \mathrm{m}^{2}$ and cold ischaemic time (CIT) below $12 \mathrm{~h}$ ) while donors beyond such criteria are either not accepted or allocated to islet cell transplantation [54-56]. However, such an approach may lead to an under-utilisation of donor pancreata which do not meet all the above criteria. Validated indices taking into account donor factors at time of donor offer and estimating graft or patient survival could aid in decisions for transplantation, particularly for donors who have borderline criteria by current standards. Also, incorporating recipient and other risk factors (also present at time of donor offer) $[33,35]$ in future risk indices may further improve their predictive ability for post-transplant outcomes. Similar risk indices (with discriminatory metrics similar to that of PDRI) incorporating both donor and recipient covariates are currently being used to guide kidney transplantation decisions both locally and abroad $[57,58]$.

Currently in Australia and New Zealand, donor race is coded differently to that of the PDRI and donor serum sodium is not routinely collected by the Australia and New Zealand Pancreas Transplant Registry. Therefore, to validate PDRI or P-PASS for use locally would require reclassification measures. Furthermore, CIT as a covariate in PDRI is not usually available at time of donor offer. Axelrod et al. acknowledge this and suggest setting the $\mathrm{CIT}$ to $12 \mathrm{~h}$ (the reference value) in such cases [3]. A similar approach would be taken with other variables such as donor ethnicity. An alternative approach is to retrospectively review local data present at time of organ offer to identify significant donor and recipient covariates associated with pancreas transplant outcomes to derive and validate a risk index which could guide local donor pancreas acceptance decisions.

\section{Conclusions}

Current data quality of studies deriving and externally validating risk indices for use in solid pancreas transplantation is inadequate. External validation for $90 \%$ of derived risk indices for solid pancreas transplantation was not performed. PDRI and P-PASS are the only risk indices currently externally validated for use in solid pancreas transplantation. PDRI was derived and validated for the outcomes of 1-year pancreas survival while P-PASS was derived and validated for donor pancreas acceptance for transplantation. Due to inadequate reporting of model performance metrics, there is currently low evidence to support their use outside current externally validated cohorts, or with different cut-offs to their derivation. To validate either risk index for use in Australia/ New Zealand would require reclassification measures due to differences in covariate coding. However, incorporating recipient and other factors which are associated with post-transplant outcomes alongside current donor covariates such as those within PDRI may increase predictive ability for future risk indices to guide solid pancreas transplantation decisions.

\section{Supplementary Information}

The online version contains supplementary material available at https://doi. org/10.1186/s12876-021-01655-2.

Additional file 1. Supplementary Files.

\section{Abbreviations}

AUROC: Area under the receiver operating characteristic curve; BMI: Body mass index; CHARMS: Critical Appraisal and Data Extraction for Systematic Reviews of Prediction Modelling Studies; CCD: Clinical Consensus Document; 
CIT: Cold ischaemic time; CVA: Cerebrovascular accident; DBD: Donation after brain death; DCD: Donation after cardiac death; EDC: Extended donor criteria; eGFR: Estimated glomerular filtration rate; EPV: Events per variable; GRADE: Grading of Recommendations, Assessment, Development and Evaluations framework; $\mathrm{H}$-L: Hosmer-Lemeshow test; HLA: Human leukocyte antigen; ICU: Intensive care unit; MESH: Medical subject headings; NP: Not performed; P: P value; PAK: Pancreas after kidney transplant; PDRI: Pancreas Donor Risk Index; P-PASS: Pre-procurement Pancreas Allocation Suitability Score; PRA: Panel-reactive antibody; PRISMA: Preferred Reporting Items for Systematic Reviews and Meta-Analyses; PROBAST: Prediction Model Risk of Bias Assessment Tool; PTA: Pancreas transplant alone; PVD: Peripheral vascular disease; R $^{2}$ : Co-efficient of determination; ROC: Receiver operating curve; SPK: Simultaneous pancreas kidney transplantation; SRTR: The Scientific Registry of Transplant Recipients; T1DM: Type 1 diabetes mellitus; T2DM: Type 2 diabetes mellitus; TRIPOD: Transparent Reporting of a multivariable prediction model for Individual Prognosis or Diagnosis; UK: United Kingdom; USA: United States of America.

\section{Acknowledgements}

Matthew Page, Research Fellow (Research Methodology, School of Public Health and Preventive Medicine, Monash University) for his valuable advice regarding systematic review structure according to Cochrane guidelines. This research was supported by an Australian Government Research Training Program (RTP) Scholarship as part of a Ph.D. degree undertaken via Monash University, Victoria, Australia.

\section{Authors' contributions}

JEHL: Study design, data collection (including title/abstract and full text screening), risk of bias analysis, narrative analysis and drafting manuscript. TC: Data collection (including title/abstract and full text screening), risk of bias analysis. KRP, JK: Supervision of narrative analysis and manuscript draft. All authors read and approved the final manuscript.

\section{Funding}

No funding was required for this study. The corresponding author is supported by a research scholarship for a Ph.D. degree via Monash University, Australia.

\section{Availability of data and materials}

The datasets used and/or analysed during the study are available from the corresponding author on reasonable request.

\section{Ethical approval and consent to participate}

Exempt from ethics approval as only previously published and non-identifiable data was required (Monash Health Local Ethics Committee).

\section{Consent for publication}

Not applicable.

\section{Competing interests}

The authors declare that they have no competing interests.

\begin{abstract}
Author details
${ }^{1}$ Department of Nephrology, Monash Medical Centre, Monash Health, 246 Clayton Road, Clayton, Melbourne, VIC 3168, Australia. ${ }^{2}$ Centre for Inflammatory Diseases, Department of Medicine, Monash University, Clayton, Melbourne, Australia. ${ }^{3}$ Department of Renal Services, Latrobe Regional Hospital, Victoria, Australia. ${ }^{4}$ Department of Epidemiology and Preventive Medicine, School of Public Health and Preventive Medicine, Monash University, Prahran, Melbourne, Australia.
\end{abstract}

Received: 21 January 2021 Accepted: 3 February 2021 Published online: 23 February 2021

\section{References}

1. Wiesner R, Edwards E, Freeman R, et al. Model for end-stage liver disease (MELD) and allocation of donor livers. Gastroenterology. 2003;124(1):91-6.
2. Rao PS, Schaubel DE, Guidinger MK, et al. A comprehensive risk quantification score for deceased donor kidneys: the kidney donor risk index. Transplantation. 2009;88(2):231-6.

3. Axelrod DA, Sung RS, Meyer KH, Wolfe RA, Kaufman DB. Systematic evaluation of pancreas allograft quality, outcomes and geographic variation in utilization. Am J Transplant. 2010;10(4):837-45.

4. Vinkers MT, Rahmel AO, Slot MC, Smits JM, Schareck WD. How to recognize a suitable pancreas donor: a Eurotransplant study of preprocurement factors. Transplant Proc. 2008;40(5):1275-8.

5. Ling JEH, Fink $M$, Westall $G$, et al. Risk indices in deceased-donor organ allocation for transplantation: review from an Australian perspective. Transplantation. 2019;103(5):875-89.

6. J DB. EuroTransplant Pancreas Allocation System (EPAS) version 5.4. 2019.

7. Kandaswamy R, Stock PG, Gustafson SK, et al. OPTN/SRTR 2018 annual data report: pancreas. Am J Transplant. 2020;20(s1):131-92.

8. Blok JJ, Kopp WH, Verhagen MJ, et al. The Value of PDRI and P-PASS as predictors of outcome after pancreas transplantation in a large European pancreas transplantation center. Pancreas. 2016:45(3):331-6.

9. Salamanca-Bustos JJ, Campos-Hernandez JP, Sánchez-Hidalgo JM, et al. Validation of the pancreatic donor risk index in simultaneous pancreaskidney transplantation performed in Córdoba Hospital from 2000 to 2015. Transplant Proc. 2016;48(9):3037-9.

10. Schenker P, Vonend O, Ertas N, Wunsch A, Viebahn R. Preprocurement pancreas allocation suitability score does not correlate with long-term pancreas graft survival. Transplant Proc. 2010;42(1):178-80.

11. Śmigielska K, Skrzypek P, Czerwiński J, et al. Usefulness of pancreas donor risk index and pre-procurement pancreas allocation suitability score: results of the Polish National Study. Ann Transplant. 2018;23:360-3.

12. Finger EB, Radosevich DM, Dunn TB, et al. A composite risk model for predicting technical failure in pancreas transplantation. Am J Transplant. 2013:13(7):1840-9.

13. Dorsey SG, Waltz CF, Brosch L, Connerney I, Schweitzer EJ, Bartlett ST. A neural network model for predicting pancreas transplant graft outcome. Diabetes Care. 1997;20(7):1128-33.

14. Clinical Guidelines for Organ Transplantation from Deceased Donors version 1.3. The Transplantation Society of Australia and New Zealand. 2019.

15. Moons KG, de Groot JA, Bouwmeester W, et al. Critical appraisal and data extraction for systematic reviews of prediction modelling studies: the CHARMS checklist. PLoS Med. 2014;11(10):e1001744.

16. Collins GS, Reitsma JB, Altman DG, Moons KG. Transparent reporting of a multivariable prediction model for individual prognosis or diagnosis (TRIPOD): the TRIPOD statement. BMJ. 2015;350:97594.

17. Ling J CT, Polkinghorne K, Kanellis J. Comparing risk indices in assessing outcomes post-pancreas transplantation: a systematic review. International Prospective Register of Systematic Reviews (PROSPERO). 2020.

18. Steyerberg EW, Moons KG, van der Windt DA, et al. Prognosis Research Strategy (PROGRESS) 3: prognostic model research. PLoS Med. 2013;10(2):e1001381.

19. Moons KGM, Wolff RF, Riley RD, et al. PROBAST: a tool to assess risk of bias and applicability of prediction model studies: explanation and elaboration. Ann Intern Med. 2019;170(1):W1-w33.

20. Wolff RF, Moons KGM, Riley RD, et al. PROBAST: a tool to assess the risk of bias and applicability of prediction model studies. Ann Intern Med. 2019;170(1):51-8

21. Steyerberg EW, Vickers AJ, Cook NR, et al. Assessing the performance of prediction models: a framework for traditional and novel measures. Epidemiology. 2010;21(1):128-38.

22. Moons KG, Kengne AP, Grobbee DE, et al. Risk prediction models: II. External validation, model updating, and impact assessment. Heart. 2012;98(9):691-8

23. Moons KG, Kengne AP, Woodward M, et al. Risk prediction models: I. Development, internal validation, and assessing the incremental value of a new (bio)marker. Heart. 2012;98(9):683-90.

24. Hosmer DW, Lemeshow S. Chapter 5: Assessing the fit of the model. In: Applied logistic regression, 2nd ed. John Wiley \& Sons, Inc; 2000. p. 143-202.

25. Kramer AA, Zimmerman JE. Assessing the calibration of mortality benchmarks in critical care: the Hosmer-Lemeshow test revisited. Crit Care Med. 2007;35(9):2052-6.

26. Steyerberg EW, Harrell FE Jr, Borsboom GJ, Eijkemans MJ, Vergouwe Y, Habbema JD. Internal validation of predictive models: efficiency 
of some procedures for logistic regression analysis. J Clin Epidemiol. 2001;54(8):774-81.

27. Royston P, Altman DG. External validation of a Cox prognostic model: principles and methods. BMC Med Res Methodol. 2013;13(1):33.

28. Balshem $H$, Helfand $M$, Schünemann $H J$, et al. GRADE guidelines: 3. Rating the quality of evidence. J Clin Epidemiol. 2011;64(4):401-6.

29. Guyatt G, Oxman AD, Sultan S, et al. GRADE guidelines: 11. Making an overall rating of confidence in effect estimates for a single outcome and for all outcomes. J Clin Epidemiol. 2013;66(2):151-7.

30. Guyatt GH, Oxman AD, Vist GE, et al. GRADE: an emerging consensus on rating quality of evidence and strength of recommendations. BMJ. 2008;336(7650):924.

31. Moher D, Liberati A, Tetzlaff J, Altman DG, The PG. Preferred reporting items for systematic reviews and meta-analyses: the PRISMA statement. PLOS Med. 2009;6(7):e1000097.

32. Grochowiecki T, Gałązka Z, Madej K, et al. Multivariate analysis of complications after simultaneous pancreas and kidney transplantation. Transplant Proc. 2014;46(8):2806-9.

33. Kasiske BL, Gustafson S, Salkowski N, et al. Optimizing the programspecific reporting of pancreas transplant outcomes. Am J Transplant. 2013;13(2):337-47.

34. Rodríguez-Villar C, Paredes D, Roque R, et al. Predictive and comparative study between clinic consensus document for pancreas acceptance and predictive value of Preprocurement Pancreas Allocation Suitability Score (P-PASS). Transplant Proc. 2019;51(2):365-8.

35. Sousa MG, Linhares MM, Salzedas-Netto AA, et al. Risk factors of pancreatic graft loss and death of receptor after simultaneous pancreas/kidney transplantation. Transplant Proc. 2014;46(6):1827-35.

36. Amaral PH, Genzini T, Perosa M, Massarollo PC. Donor risk index does not predict graft survival after pancreas transplantation in Brazil. Transplant Proc. 2015;47(4):1025-8.

37. Ayami MS, Grzella S, Kykalos S, Viebahn R, Schenker P. Pancreas donor risk index but not pre-procurement pancreas allocation suitability score predicts pancreas graft survival: a cohort study from a Large German Pancreas Transplantation Center. Ann Transplant. 2018;23:434-41.

38. Franz C, Görtz M, Wührl M, et al. The role of Pre-Procurement Pancreas Suitability Score (P-PASS) and Pancreas Donor Risk Index (PDRI) in the outcome of simultaneous pancreas and kidney or pancreas after kidney transplantation. Ann Transplant. 2019;24:439-45. https://doi.org/10.12659 /aot.915852.

39. Horvath SKU, Ilham MA, Ablorsu E, Elker D, Asderakis A. How useful are the US PDRI and the European P-PASS scores in predicting outcomes in UK pancreas transplant patients. Transpl Int. 2015;28:140.

40. Kopp WH, de Vries E, de Boer J, et al. Donor risk indices in pancreas allocation in the Eurotransplant region. Transpl Int. 2016;29(8):921-9.

41. Mittal S, Sharples E, Lee F, et al. App to reality: snapshot validation of the US Pancreas Donor Risk Index in a UK center. J Surg Res. 2013;183(2):841-5.

42. Mittal S, Lee FJ, Bradbury L, et al. Validation of the Pancreas Donor Risk Index for use in a UK population. Transpl Int. 2015;28(9):1028-33.

43. Foltys DB, Kaths JM, Zimmermann T, Heise M, Hoppe-Lotichius M, Otto G. Ten years of simultaneous pancreas-kidney transplantation: a retrospective single-center analysis of prospectively obtained data. Transplant Proc. 2011:43(9):3267-9.

44. Garcia-Roca RSG, Del Gobbo GD, Sanchez-Cabus S, Paredes D, Ricart MJ. Impact of pancreas donor scoring system in graft complications and outcomes: single center experience. Transpl Int. 2011;24(s2):4.
45. Lan LMW, Chaal P, Marion V, Opdam HI, Saunder A, Kanellis J. Factors influencing the acceptance of donor solid-organ pancreata for transplantation. An Australian single centre review. Transplantation. 2010;90:226.

46. Ziaja J, Król R, Pawlicki J, et al. Donor-dependent risk factors for early surgical complications after simultaneous pancreas-kidney transplantation. Transplant Proc. 2011;43(8):3092-6.

47. Woeste G, Moench C, Hauser IA, Geiger H, Scheuermann E, Bechstein WO Can the preprocurement pancreas suitability score predict ischemiareperfusion injury and graft survival after pancreas transplantation? Transplant Proc. 2010;42(10):4202-5.

48. Bouwmeester W, Zuithoff NP, Mallett $\mathrm{S}$, et al. Reporting and methods in clinical prediction research: a systematic review. PLoS Med. 2012;9(5):1-12.

49. Heus P, Damen J, Pajouheshnia R, et al. Poor reporting of multivariable prediction model studies: towards a targeted implementation strategy of the TRIPOD statement. BMC Med. 2018;16(1):120.

50. Vinkers MTRA, Slot MC, Smits JM, Schareck WD. Influence of a donor quality score on pancreas transplant survival in the Eurotransplant area. Transplant Proc. 2008;40:3606-8.

51. Rudolph EN, Dunn TB, Mauer D, et al. HLA-A, -B, -C, -DR, and -DQ matching in pancreas transplantation: effect on graft rejection and survival. Am J Transplant. 2016;16(8):2401-12.

52. Hilling DE, Baranski AG, Haasnoot A, van der Boog PJ, Terpstra OT, Marang-van de Mheen PJ. Contribution of donor and recipient characteristics to short- and long-term pancreas graft survival. Ann Transplant. 2012;17(4):28-38.

53. Cook NR. Use and misuse of the receiver operating characteristic curve in risk prediction. Circulation. 2007;115(7):928-35.

54. Fridell JA, Rogers J, Stratta RJ. The pancreas allograft donor: current status, controversies, and challenges for the future. Clin Transplant. 2010;24(4):433-49.

55. Maglione M, Ploeg RJ, Friend PJ. Donor risk factors, retrieval technique, preservation and ischemia/reperfusion injury in pancreas transplantation. Curr Opin Organ Transplant. 2013;18(1):83-8.

56. Stratta RJ, Gruessner AC, Gruessner RWG. Chapter 31-Pancreas transplantation: current issues, unmet needs, and future perspectives. In: Orlando G, Piemonti L, Ricordi C, Stratta RJ, Gruessner RWG, editors. Transplantation, bioengineering, and regeneration of the endocrine pancreas. London: Academic Press; 2020. p. 375-86.

57. Clayton PA, Dansie K, Sypek MP, et al. External validation of the US and UK kidney donor risk indices for deceased donor kidney transplant survival in the Australian and New Zealand population. Nephrol Dial Transplant. 2019;34(12):2127-31.

58. Clayton PA, McDonald SP, Snyder JJ, Salkowski N, Chadban SJ. External validation of the estimated posttransplant survival score for allocation of deceased donor kidneys in the United States. Am J Transplant. 2014;14(8):1922-6.

\section{Publisher's Note}

Springer Nature remains neutral with regard to jurisdictional claims in published maps and institutional affiliations.

Ready to submit your research? Choose BMC and benefit from:

- fast, convenient online submission

- thorough peer review by experienced researchers in your field

- rapid publication on acceptance

- support for research data, including large and complex data types

- gold Open Access which fosters wider collaboration and increased citations

- maximum visibility for your research: over $100 \mathrm{M}$ website views per year

At BMC, research is always in progress.

Learn more biomedcentral.com/submissions 